\title{
Bioinformatics Analysis of the Lycopene $\beta$-Cyclase Gene in Cabbage (Brassica oleracea var. capitata)
}

\author{
Bo Sun, Min Jiang, Qiao Yuan, Fen Zhang, and Haoru Tang* \\ College of Horticulture, Sichuan Agricultural University, Chengdu 611130, China. \\ *Correspondence author, E-mail addresses: htang@sicau.edu.cn.
}

Keywords: Cabbage, carotenoids, Lycopene $\beta$-cyclase (LCYb), bioinformatics analysis.

\begin{abstract}
Lycopene $\beta$-cyclase (LCYb) is an important enzyme in carotenoid biosynthesis. Here, the Brassica oleracea var. capitata $L C Y b(B o c L C Y b)$ gene sequence was obtained from Brassica database (BRAD), and preformed for bioinformatics analysis. The BocLCYb gene mapped to Scaffold000212, and contains an open reading frame of 1,500 bp that encodes a 499 -amino acid protein with a calculated molecular mass of $50.90 \mathrm{kD}$ and an isoelectric point (pI) of 6.77. Subcellular localization predicted the BocLCYb gene was in the cytoplasm and nucleus. The conserved domain of the BocLCYb protein is PLNO2463. The BocLCYb protein is most closely related to Raphanus sativus. The findings of the present study provide a molecular basis for the elucidation of $L C Y b$ gene function in cabbage.
\end{abstract}

\section{Introduction}

Cabbage (Brassica oleracea var. capitata) is a member of the Brassicaceae family that is widely distributed in the world. In China, cabbage is an important vegetable crop, and consumed considerable every years. Cabbage is generally grown for its leafy head as common edible part, which are crispy, tender, and tasty [1]. Besides its good flavor, cabbage is also a rich source of nutrients, antioxidants, and anticarcinogenic compounds, including carbohydrates, vitamin C, carotenoids, and glucosinolates [1-2].

Carotenoids, which are synthesized in various photosynthetic and non-photosynthetic organisms, including algae, plants, and some bacteria and fungi, are a class of 40-carbon hydrocarbon compounds derived from a terpenoid precursor [3]. The enzymes involved in the carotenoid biosynthetic pathway have been extensively studied in various plants, including Arabidopsis [4], tomato [5], and citrus [6]. The first key step in carotenoid biosynthesis involves the production of a 40-carbon phytoene from two geranylgeranyl pyrophosphate (GGPP) molecules, which is catalyzed by phytoene synthase (PSY) [7-8]. Then, lycopene (colored carotenoid) is converted from phytoene (non-color carotenoid) by desaturases and isomerases, including phytoene desaturases (PDS) [9],

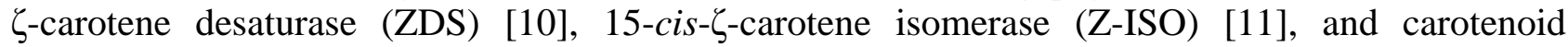
isomerase (CRTISO) [4]. Hereafter, bifurcation of the carotenoid biosynthetic pathway occurs, and the production of $\beta$-carotene and $\alpha$-carotene is catalyzed by lycopene $\beta$-cyclase (LCYb) and lycopene $\varepsilon$-cyclase (LCYe) [12-13].

$\mathrm{LCYb}$ is essential for the cyclization of lycopene to form $\beta$-ring cyclic end groups [3].The genes encoding the LCYb protein have been isolated in various plant species, including Arabidopsis, tomato, watermelon and Hibiscus esculentus [14]. To date, research studies on $L C Y b$ in cabbage are limited. In the present study, the $L C Y b$ gene sequence of cabbage was obtained from web database, and then bioinformatics analysis of the $L C Y b$ gene were analyzed. The present study aimed to establish the foundation for further studies on the molecular mechanism of $L C Y b$ in cabbage.

\section{Materials and methods}

\subsection{Sequence Obtain of the BocLCYb Gene.}

The genomic DNA and mRNA sequences of $L C Y b$ gene of cabbage were downloaded and 
obtained from The Brassica database (BRAD) (http://brassicadb.org), and then used to subsequent bioinformatic analysis.

\subsection{Bioinformatics Analysis of the BocLCYb Gene.}

The amino acid sequence, protein molecular weight, isoelectric point, stability index, and hydrophobicity of the BocLCYb gene were analyzed and predicted by ExPASy (http://web.expasy.org) and NCBI (https://www.ncbi.nlm.nih.gov/). Subcellular localization was predicted by WoLF PSORT (http://www.genscript.com/wolf-psort.html). The conserved domain were predicted by NCBI (https://www.ncbi.nlm.nih.gov/Structure/cdd/wrpsb.cgi). Phylogenetic tree analysis of the LCYb proteins was executed in MEGA 6.0 using the neighbor-joining (NJ) method.

\section{Results}

\subsection{Analysis on Genomic Organization.}

The Brassica database (BRAD) was used to analyze the chromosomal localization and genomic organization of BocLCYb. The gene ID in BRAD is Bol011368. The BocLCYb gene was mapped to Scaffold000212 and has 1 exon and 0 intron (Fig. 1).

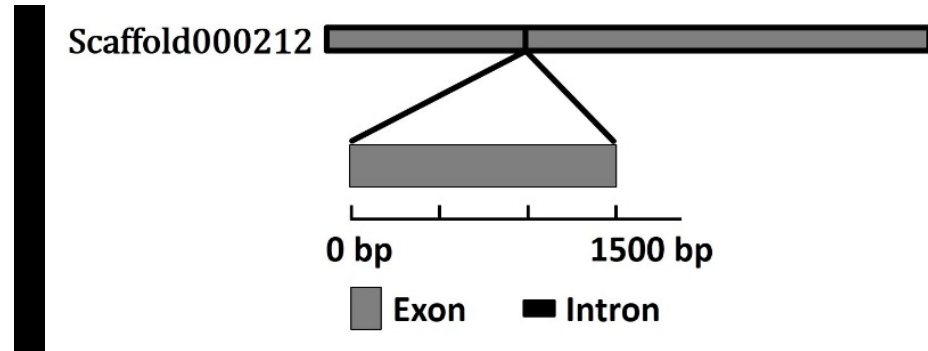

Fig. 1 Chromosomal location and genomic structure of BocLCYb.

\subsection{Protein Physical and Chemical Properties Analysis.}

Sequence analysis indicated that the $B o c L C Y b$ gene contained a 1,500-bp open reading frame (ORF), which encoded a 499-amino acids protein with a calculated molecular mass of $55.90 \mathrm{kD}$ and an isoelectric point (pI) of 6.77. The amino acid types and proportions of the BocLCYb gene was shown in Figure 2, the highest number of amino acid is Leucine (Leu), whereas the lowest number is Cysteine (Cys) and Tryptophan (Trp). Its predicted formula was $\mathrm{C}_{2506} \mathrm{H}_{3947} \mathrm{~N}_{683} \mathrm{O}_{724} \mathrm{~S}_{21}$. Its total average hydrophilicity index was -0.152 , liposoluble index was 90.96, and instability index in solution was 35.58 .

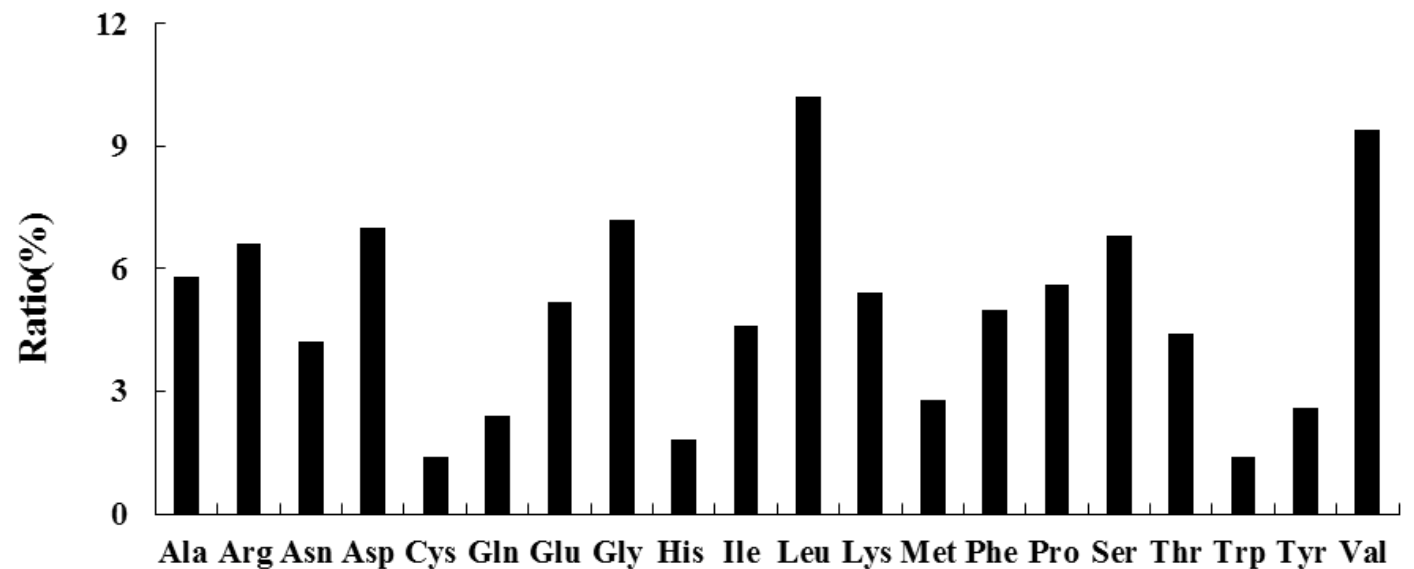

Amino Acid

Fig. 2 Amino acid composition of BocLCYb 


\subsection{Subcellular Localization and Conserved Domain Analysis.}

Subcellular localization of the BocLCYb gene was predicted by WoLF PSORT to be in the cytoplasm and nucleus. The analysis using Conserved Domain Database (CDD) demonstrated that the amino acid sequence of the BocLCYb protein has one conserved domain PLN02463 and one NADB_Rossmann superfamily.

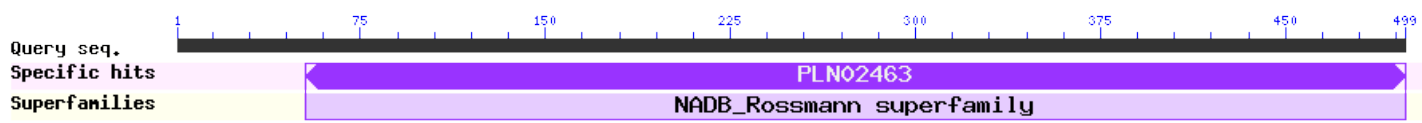

Fig. 3 Conserved domains analysis of BocLCYb

\subsection{Homology and Phylogenetic Tree Analysis.}

A phylogenetic tree was constructed to illustrate the relationship among the LCYb proteins of cabbage and 19 other higher plant species (Fig. 4). A total of three major clusters were identified. Sequence alignment indicated that the BocLCYb protein is more closely related to Raphanus sativus, which belonged to the Cruciferae branch.

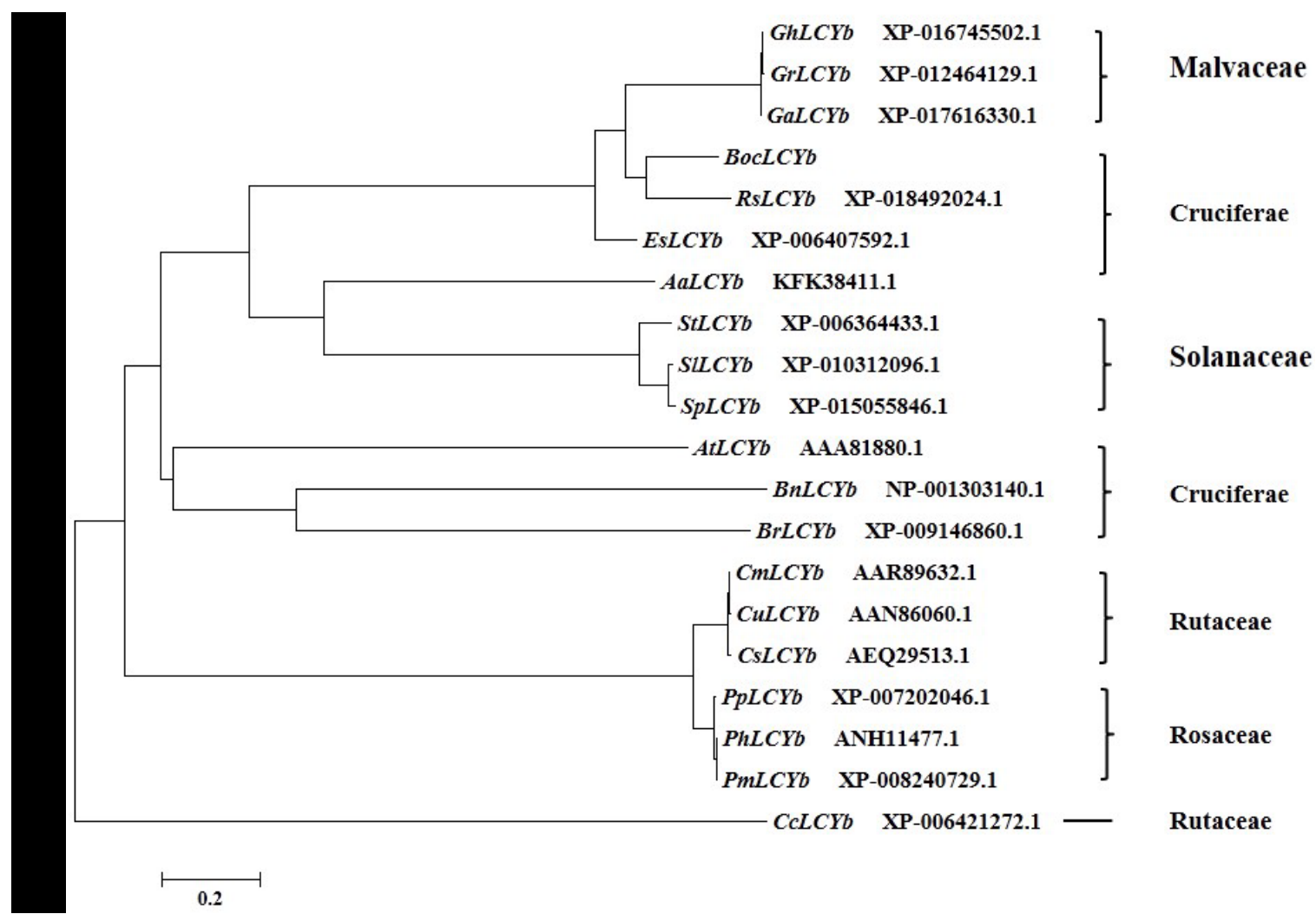

Fig. 4 Phylogenetic tree analysis of BocLCYb and LCYb proteins of other species

\section{Discussion}

The present study analyzed the BocLCYb gene of cabbage. LCYb enzyme is encoded by a single -copy genes in Hibiscus esculentus [14]. While, the $L C Y b$ gene occurred as a single copy in cabbage, indicating that the enzyme may have undergone similar evolutionary patterns. Previous studies have shown that the LCYb protein is relatively conserved in plants [14]. The LCYb protein of Hibiscus esculentus is similar to the LCYb protein of Gossypium hirsutum, showing 91\% homology [14]. The findings of the present study show that LCYb from cabbage is highly conserved, particularly in the Cruciferae, similar to that observed in earlier reports. The findings of the present study may serve as a foundation for future studies on the functions of LCYb in carotenoid metabolism in cabbage. 


\section{Acknowledgements}

This work was supported by National Natural Science Foundation of China (31500247), key project of Department of Education of Sichuan Province (14ZA0016), and National Student Innovation Training Program (201710626030).

\section{References}

[1] M. Wennberg, J. Ekvall, K. Olsson, and M. Nyman, Changes in carbohydrate and glucosinolate composition in white cabbage (Brassica oleracea var. capitata) during blanching and treatment with acetic acid, Food Chem. 95 (2006) 226-236.

[2] S. Rokayya, C.J. Li, Y. Zhao, Y. Li, and C.H. Sun, Cabbage (Brassica oleracea L. var. capitata) phytochemicals with antioxidant and anti-inflammatory potential, Asian Pac. J. Cancer Prev. 14 (2014) 6657-6662.

[3] N. Nisar, L. Li, S. Lu, N.C. Khin, and B.J. Pogson, Carotenoid metabolism in plants, Mol. Plant 8 (2015) 68-82.

[4] H. Park, S.S. Kreunen, A.J. Cuttriss, D. Della Penna, and B.J. Pogson, Identification of carotenoid isomerase provides insight into carotenoid biosynthesis, prolamellar body formation, and photomorphogenesis, Plant Cell 14 (2002) 321-332.

[5] T. Isaacson, G. Ronen, D. Zamir, and J. Hirschberg, Cloning of tangerine from tomato reveals a carotenoid isomerase essential for the production of b-carotene and xanthophylls in plants, Plant Cell 14 (2002) 333-342.

[6] M. Kato, Y. Ikoma, H. Matsumoto, M. Sugiura, H. Hyodo, and M. Yano, Accumulation of carotenoids and expression of carotenoid biosynthetic genes during maturation in critrus fruit, Plant Physiol. 134 (2004) 824-837.

[7] P.A. Scolnik, and G.E. Bartley, Nucleotide sequence of an Arabidopsis cDNA for phytoene synthase, Plant Physiol. 104 (1994) 1471-1472.

[8] P.R. Li, S.J. Zhang, S.F. Zhang, F. Li, H. Zhang, F. Cheng, J. Wu, X.W. Wang, and R.F. Sun, Carotenoid biosynthetic genes in Brassica rapa: comparative genomic analysis, phylogenetic analysis, and expression profiling, BMC Genomics 16 (2015) 492.

[9] P.A. Scolnik, and G.E. Bartley, Phytoene desaturase from Arabidopsis, Plant Physiol. 103 (1993) 1475.

[10]P.A. Scolnik, and G.E. Bartley, Nucleotide sequence of zeta-carotene desaturase (accession no. U38550) from Arabidopsis, Plant Physiol. 109 (1995) 1499.

[11]Y. Chen, F. Li, and E.T. Wurtzel, Isolation and characterization of the Z-ISO gene encoding a missing component of carotenoid biosynthesis in plants, Plant Physiol. 153 (2010) 66-79.

[12]B. Pogson, K.A. McDonald, M. Truong, G. Britton, and D. Della Penna, Arabidopsis carotenoid mutants demonstrate that lutein is not essential for photosynthesis in higher plants, Plant Cell 8 (1996) 1627-1639.

[13]Y.M. Shi, R. Wang, Z.P. Luo, L.F. Jin, P.P. Liu, Q.S. Chen, Z.F. Li, F. Li, C. Y. Wei, M.Z. Wu, P. Wei, H. Xie, L.B. Qu, F.C. Lin, and J. Yang, Molecular cloning and functional characterization of the lycopene $\varepsilon$-cyclases gene via virus-induced gene silencing and its expression pattern in Nicotiana tabacum, Int. J. Mol. Sci. 15 (2014) 14766-14785.

[14] Y.P. Li, M.D. Chen, H.S. Zhu, Q.F. Wen, J.T. Liu, Cloning and expression analysis of lycopene beta-cyclase gene in Hibiscus esculentus L. J. Plant Genetic Resources 19 (2018) 168-177. 